\title{
Rahmen für eine Vereinbarung nach § 3 Absatz 2 Satz 3 des Transfusionsgesetzes (TFG) zwischen den Spendeeinrichtungen in der Bundesrepublik Deutschland
}

\author{
Jürgen Bux ${ }^{a}$ Helmut Frenzel $^{a} \quad$ Erhard Seifried $^{b}$ Manfred Stähle $^{b, d, f}$ Thomas Müller $^{c}$ \\ Rainer Waßmann ${ }^{c}$ Wolfgang Rüstig ${ }^{d}$ Franz Weinauer ${ }^{e} \quad$ Georg Götz $^{\mathrm{e}} \quad$ Axel Huck $^{\dagger}$ \\ Wolfgang Stangenberg ${ }^{g}$ Helmut Kreuzer ${ }^{g} \quad$ Walter Hitzler $^{\mathrm{h}} \quad$ Gabriele Hutschenreuter $^{\mathrm{h}}$ \\ Knud-Peter Krause ${ }^{i}$ Katrin Scholz ${ }^{i}$ Marcell Heim ${ }^{j} \quad$ Kirsten Seidel ${ }^{j}$ Michael Brod $^{k}$ \\ Volker Kiefel' Hans-Günter Hennecke ${ }^{m}$
}

\footnotetext{
${ }^{a}$ Deutsches Rotes Kreuz - Blutspendedienst West gGmbH der Ländesverbände Nordrhein, Westfalen-Lippe, Rheinland-Pfalz und Saarland ${ }^{\mathrm{b}}$ Deutsches Rotes Kreuz - Blutspendedienst Baden-Württemberg - Hessen gGmbH

'Blutspendedienst der Deutsches Rotes Kreuz-Landesverbände Niedersachsen, Sachsen-Anhalt, Thüringen, Oldenburg und Bremen gGmbH

${ }^{\mathrm{d}}$ Deutsches Rotes Kreuz - Blutspendedienst Ost gGmbH für die Bundesländer Berlin, Brandenburg und Sachsen

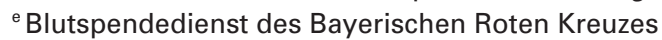

${ }^{\mathrm{f}}$ Deutsches Rotes Kreuz - Blutspendedienst Nord gGmbH für die Bundesländer Hamburg und Schleswig-Holstein

${ }^{9}$ Deutsches Rotes Kreuz - Blutspendedienst Mecklenburg-Vorpommern gGmbH

${ }^{\text {h }}$ Arbeitsgemeinschaft der Ärzte staatlicher und kommunaler Bluttransfusionsdienste (StKB)

' Verband unabhängiger Blutspendedienste e.V.

${ }^{\mathrm{A}}$ ARGE Plasmapherese

${ }^{k}$ Blutspendedienst der Bundeswehr, Wehrbereichsverwaltung Süd

' Deutsche Gesellschaft für Transfusionsmedizin und Immunhämatologie (DGTI)

${ }^{\mathrm{m}}$ Deutscher Landkreistag
}

\section{Präambel}

1) Das Blutspendewesen in der Bundesrepublik Deutschland genießt ein hohes Ansehen und Vertrauen in der Bevölkerung. Die Spendeeinrichtungen sind deshalb in besonderem Maße dem Blutspender verpflichtet, der sein Blut für schwerkranke Patienten uneigennützig spendet.

2) Spendeeinrichtungen im Sinne dieser Vereinbarung sind Einrichtungen, die Spenden entnehmen oder deren Tätigkeit auf die Entnahme von Spenden und, soweit diese zur Anwendung bestimmt sind, auf deren Testung, Verarbeitung, Lagerung und das Inverkehrbringen gerichtet ist ( $\S$ 2 Nr. 2 TFG)

3) Blutprodukte im Sinne dieser Vereinbarung sind Blutzubereitungen im Sinne des $\S 4$ Absatz 2 des Arzneimittelgesetzes, Sera aus menschlichem Blut im Sinne des $\$ 4$ Absatz 3 des Arzneimittelgesetzes und Blutbestandteile, die zur Herstellung von Wirkstoffen oder Arzneimitteln bestimmt sind ( $\$ 2$ Nr. 3 TFG).

4) Blutkomponenten im Sinne dieser Vereinbarung sind zelluläre Blutprodukte wie Erythrozytenkonzentrate,
Thrombozytenkonzentrate, Granulozytenkonzentrate sowie therapeutisches Frischplasma.

5) Einrichtungen der Krankenversorgung im Sinne dieser Vereinbarung sind Krankenhäuser und andere ärztliche Einrichtungen, die Personen behandeln ( $\$ 14$ Absatz 2 Satz 3 TFG).

6) Im Blutspendewesen sind in Deutschland gegenwärtig tätig:

a) die Rotkreuz-Blutspendedienste,

b) die staatlichen und kommunalen

Bluttransfusionsdienste,

c) die privaten Blutspendedienste,

d) die Spendeeinrichtungen der Plasma verarbeitenden Industrie und

e) der Blutspendedienst der Bundeswehr.

7) Einrichtungen der Krankenversorgung ohne eigene Spendeeinrichtungen werden überwiegend von den RotkreuzBlutspendediensten und anderen überregionalen Spendeeinrichtungen mit den notwendigen Blutkomponenten und teilweise auch mit den zugehörigen transfusionsmedizinischen Leistungen versorgt. Des Weiteren sichern

\section{KARGER}

Fax +497614520714

Information@Karger.de

www.karger.com (c) 2010 S. Karger GmbH, Freiburg 
diese Spendeeinrichtungen für Einrichtungen der Krankenversorgung ohne eigene Spendeeinrichtungen teilweise die Versorgung mit speziellen Blutprodukten und Dienstleistungen entsprechend der einrichtungsspezifischen Aufgaben.

8) Einrichtungen der Krankenversorgung mit eigenen Spendeeinrichtungen sind für die Versorgung mit Blutkomponenten, transfusionsmedizinischen Leistungen und für das transfusionsmedizinische Qualitätsmanagement ihrer Einrichtung verantwortlich und versorgen je nach Standort auch umliegende Einrichtungen der Krankenversorgung. Diese lokalen und teilweise auch regionalen Spendeeinrichtungen sichern über die Grundversorgung mit Blutkomponenten hinaus die Versorgung mit speziellen Blutprodukten, die sich aus speziellen Aufgaben ergeben.

9) Die Spendeeinrichtungen der Plasma verarbeitenden Industrie gewinnen hauptsächlich Plasma zur Fraktionierung.

10) Die Unterzeichner bekräftigen die Überzeugung, dass sie diese bewährte Struktur des Blutspendewesens für ausreichend flexibel und anpassungsfähig halten, um künftigen Herausforderungen gerecht werden zu können.

11) § 3 TFG enthält einen Versorgungsauftrag, der an die Spendeeinrichtungen gerichtet ist. $\S 3$ Absatz 2 Satz 1 TFG verpflichtet die Spendeeinrichtungen zur Zusammenarbeit. Nach § 3 Absatz 2 Satz 3 TFG haben sie die Einzelheiten der Zusammenarbeit in einer Vereinbarung festzulegen.

Mit der vorliegenden Vereinbarung folgen die Spendeeinrichtungen diesem gesetzlichen Auftrag.

\section{Artikel I - Versorgung von Einrichtungen der Krankenversorgung ohne eigene Spendeeinrichtungen}

1) Die Spendeeinrichtungen haben die Aufgabe, die Patientenversorgung mit Blut und Blutprodukten aus Blut- und Plasmaspenden sicherzustellen.

2) Die Spendeeinrichtungen verpflichten sich, an 7 Tagen in der Woche rund um die Uhr die Abgabe von Blutkomponenten zu gewährleisten und die Durchführung aller für die Transfusion notwendigen, immunhämatologischen Untersuchungen sicherzustellen.

\section{Artikel II - Zusammenarbeit der Spendeeinrichtungen}

1) Die Spendeeinrichtungen gewährleisten einen fairen Wettbewerb im Rahmen des bestehenden Blutspendewesens.

2) Die Spendeeinrichtungen verpflichten sich auf der Grundlage der Muster-Berufsordnung für die deutschen Ärztinnen und Ärzte zur kollegialen und vertrauensvollen $\mathrm{Zu}$ sammenarbeit im Rahmen ihrer Versorgungsleistungen.
3) Die Spendeeinrichtungen arbeiten zusammen

a) in Fachgesellschaften, Berufsverbänden und Standesorganisationen sowie im Arbeitskreis Blut des Bundesministeriums für Gesundheit

i) bei der Entwicklung und Umsetzung neuer Konzepte zur Spendergewinnung,

ii) zum Erhalt der Wiederholungsspender,

iii) bei der ständigen Weiterentwicklung der Qualität der Blutprodukte nach dem Stand von Wissenschaft und Technik,

iv) bei der Fortentwicklung der transfusionsmedizinischen und hämostaseologischen Patientenversorgung und

v) bei der Umsetzung und Weiterentwicklung der nationalen und internationalen Standards bei der Herstellung und Anwendung von Blutprodukten;

b) in der Versorgung von Einrichtungen der Krankenversorgung bei Versorgungsengpässen oder in Notfallsituationen. In solchen Situationen unterstützen sich die Spendeeinrichtungen untereinander.

4) Die Spendeeinrichtungen verpflichten sich, mit den aus in Deutschland gewonnenen Blut und Blutbestandteilen hergestellten Blutkomponenten vorrangig die Nachfrage in Deutschland zu befriedigen; dies ist in entsprechenden Versorgungsverträgen zu berücksichtigen.

5) Versorgung mit Blutkomponenten im Regelfall

a) Die Spendeeinrichtungen wirken darauf hin, die Regelversorgung mit Blutkomponenten durch die Spendeeinrichtungen entsprechend ihrer jeweiligen Erfordernisse sicherzustellen. Der Versorgungsbedarf mit Blutkomponenten im Regelfall soll im Voraus zwischen den Spendeeinrichtungen abgestimmt werden. Dazu können die Spendeeinrichtungen insbesondere Lieferverträge mit einer oder mehreren anderen Spendeeinrichtungen über die in der Vertragslaufzeit zu liefernde Menge von Blutkomponenten unter Berücksichtigung der Blutgruppenverteilung in der Bevölkerung abschließen. Die Vertragslaufzeit soll 12 Monate nicht über- oder unterschreiten. In jedem Vertrag zur verbindlichen Lieferung/ Abnahme von Blutkomponenten ist ein Spielraum bezüglich des Lieferrahmens für Fälle von unerwartetem, vorübergehendem Mehr- oder Minderbedarf vorzusehen.

b) Alle Spendeeinrichtungen wirken darauf hin, dass Blutkomponenten mit den Blutgruppen entsprechend der Blutgruppenverteilung in der Bevölkerung zum Einsatz kommen.

6) Versorgung mit Blutkomponenten bei Versorgungsengpässen

a) Versorgungsengpässe treten auf, wenn der Bedarf an Blutkomponenten vorübergehend nicht durch die versorgenden Spendeeinrichtungen abgedeckt werden kann. Dies kann insbesondere bedingt sein durch: 
i) unvorhergesehenen Spendenrückgang,

ii) die Spezifität der aktuellen Anforderung oder

iii) akuten Mehrbedarf an Blutkomponenten.

b) In solchen Situationen helfen sich die Spendeeinrichtungen untereinander aus. Hierbei ist die spezielle Versorgungssituation jeder Spendeeinrichtung zu beachten.

7) Versorgung mit Blutkomponenten in Notfallsituationen

a) Eine Notfallsituation im Sinne dieser Vereinbarung tritt ein, wenn ohne Unterstützung durch fremde Spendeeinrichtungen ein Patient infolge Blutkomponentenmangels lebensbedrohlich gefährdet würde.

b) In Notfallsituationen müssen die um Hilfe gebetenen Spendeeinrichtungen unverzüglich die angeforderten Blutkomponenten liefern. Die Lieferung erfolgt AB0identisch und in der Regel Rh(D) positiv. Die Lieferung in Notfallsituationen darf nicht an Bedingungen geknüpft werden (z.B. an die zusätzliche Abnahme von nicht benötigten Blutgruppen).

c) Den um Hilfe gebetenen Spendeeinrichtungen sind anonymisierte klinische Daten des Notfallpatienten, insbesondere seine Blutgruppe einschließlich des RhesusFaktors, sowie die Notfallursache anzugeben.

d) Zur Vermeidung von zeitlichen Verzögerungen können die um Hilfe gebetenen Spendeeinrichtungen in Notfallsituationen die zu versorgende Einrichtung der Krankenversorgung direkt beliefern.

8) Bei begründeten Zweifeln an der sachgerechten Durchführung des Bestellverfahrens von Blutkomponenten oder am Abgabeverhalten der Spendeeinrichtung kann sowohl im Regelfall als auch im Nachhinein bei Versorgungsengpässen oder Notfallsituationen die Beratungskommission Blutversorgung (Art. V dieser Vereinbarung) angerufen werden.

\section{Artikel III - Werbung und Blutspendeaktionen}

1) Die Werbung der Spendeeinrichtungen soll die besondere Stellung des Blutspendewesens in der öffentlichen Meinung berücksichtigen. Die Spendeeinrichtungen verpflichten sich, ihre Werbung sachlich zu gestalten und eine Diskreditierung anderer Spendeeinrichtungen in der Öffentlichkeit zu vermeiden.

2) Die Spendeeinrichtungen verpflichten sich, Blutspender von anderen Spendeeinrichtungen nicht gezielt abzuwerben. Die Spendeeinrichtungen wirken darauf hin, dass Blutspender in der Regel nicht in mehreren Spendeeinrichtungen gleichzeitig zum Spenderstamm gehören.

3) Blutspendetermine können in den Medien bekannt gegeben werden. Spendeeinrichtungen, die im Einzugsgebiet anderer Spendeeinrichtungen außerplanmäßige Spendeaktionen durchführen, sollen diese Spendeeinrichtungen im Voraus darüber informieren.

\section{Artikel IV - Blutspendedienst der Bundeswehr}

1) Der Blutspendedienst der Bundeswehr versorgt sanitätsdienstliche Einrichtungen im In- und insbesondere im Ausland mit Blutzubereitungen. Im Rahmen freier Kapazitäten können auch zivile Einrichtungen versorgt werden.

2) Im Geschäftsbereich des Bundesministeriums der Verteidigung finden die Vorschriften des TFG entsprechende Anwendung.

3) Der Blutspendedienst der Bundeswehr trägt die Vereinbarung mit, soweit sein Auftrag (Versorgung der Einsatzkontingente der Bw) dem nicht entgegensteht.

4) Die zur Transfusion notwendigen immunhämatologischen Untersuchungen werden durch die zu versorgenden Bundeswehrkrankenhäuser bzw. Einsatzlazarette in eigener Zuständigkeit durchgeführt, weshalb eine Dienstbereitschaft im obigen Sinne für den Blutspendedienst der Bundeswehr entfällt.

\section{Artikel V - Beratungskommission Blutversorgung}

1) Treten zwischen den Spendeeinrichtungen Streitigkeiten auf, die mit der Blutversorgung in Deutschland zusammenhängen, insbesondere

a) konkrete Streitfälle zwischen Spendeeinrichtungen bezüglich der Blutversorgung im Regelfall, bei Versorgungsengpässen oder in Notfallsituationen gemäß Art. II Ziffer 8 oder

b) konkrete oder abstrakte Streitfragen im Hinblick auf Werbemaßnahmen der Spendeeinrichtungen kann eine Beratungskommission Blutversorgung angerufen werden.

2) Die Beratungskommission Blutversorgung setzt sich aus Vertretern der beteiligten Spendeeinrichtungen und jeweils einem Vertreter

a) der Rotkreuz-Blutspendedienste,

b) der Arbeitsgemeinschaft der Ärzte staatlicher und kommunaler Bluttransfusionsdienste e.V. (StKB),

c) des Verbandes unabhängiger Blutspendedienste (VUBD),

d) der Arbeitsgemeinschaft Plasmapherese (ARGE Plasmapherese) und

e) des Blutspendedienstes der Bundeswehr zusammen. Der Vorsitz wird fallweise der Bundesärztekammer angetragen.

3) Die Beratungskommission Blutversorgung entscheidet einvernehmlich. Die Entscheidung sowie der Inhalt der Gespräche sind streng vertraulich. Die beteiligten Parteien verpflichten sich, die einvernehmliche Entscheidung künftig zu beachten.

4) Ihre für einen Termin entstehenden Sach- und Reisekosten tragen die Beteiligten und Mitglieder jeweils selbst. 


\section{Deutscher \\ Städtetag}

Deutscher Städtetag, Postfach 5106 20, D-50942 Köln

Deutscher Stådte- und Gemeindebund, Marienstraße 6, 12207 Berlin

Herrn

Friedger von Auer

Bundesministerium für Gesundheit

Heilsbachstraße 18

53123 Bonn

E-Mail: friedger:vonauer a bmg.bund.de

\section{DStGB \\ A $\mathbf{A}$ Dentscher Strodte.}

$24.03 .2009 / \mathrm{Fa}$.

Bearbeitet von

Andrea Vontz-Liesegang

Telefon + $492213771-260$

Telefax + $492213771-177$

E-Mail:

andrea.vontz@staedtetag.de

Aktenzeichen

53.08.09

Vereinbarung nach $\$ 3$ Abs. 2 Satz 3 des Transfusionsgesetzes (TFG)

Sehr geehrter Herr von Auer,

wie besprochen, können der Deutscher Städtetag und der Deutsche Städte- und Gemeindebund die Vereinbarung der Spendeeinrichtungen aus den bereits geschilderten formalen Gründen nicht unterzeichnen. Gerne kommen wir allerdings Ihrer Bitte nach, eine Protokollerklärung mit dem Wortlaut "Der Deutsche Städtetag und der Deutsche Städte- und Gemeindebund sind Unterstützer der Vereinbarung." abzugeben. Dadurch wird deutlich, dass wir die Inhalte der Vereinbarung der Spendeeinrichtungen unterstützen.

Mit freundlichen Grüßen

In Vertretung

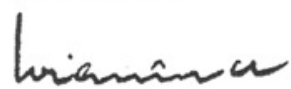

Dr. Manfred Wienand

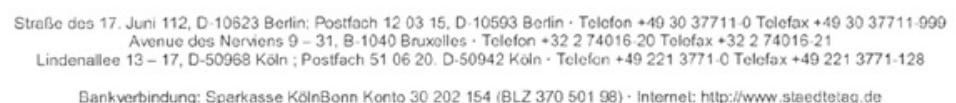

Abb. 1. Deutscher Städtetag - Zustimmung zur Vereinbarung § 3 Absatz 2 Satz 3 TFG. 


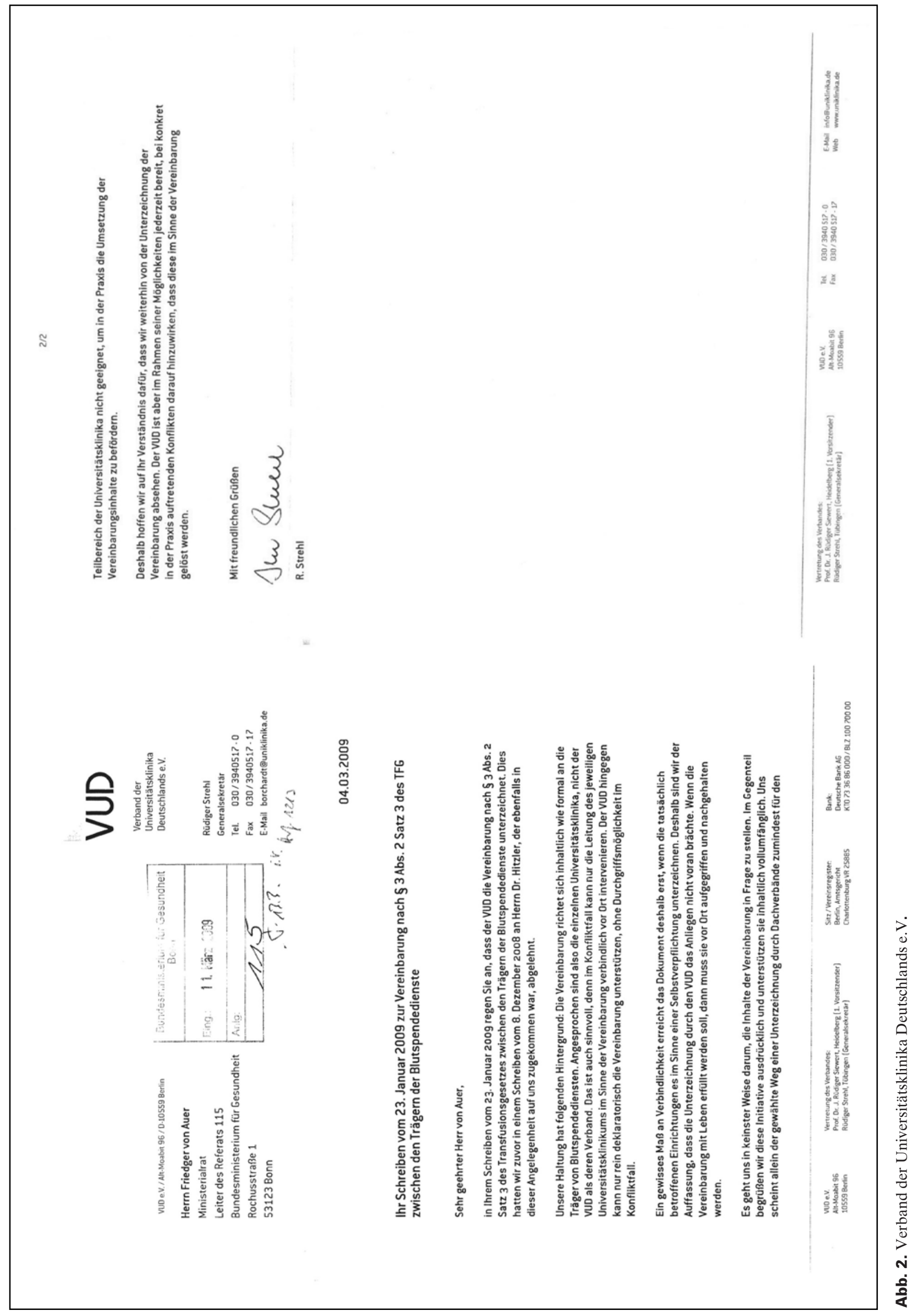




\section{Artikel VI - Schlussbestimmungen}

Die Unterzeichner verpflichten sich, in ihrem Zuständigkeitsbereich den aufgeführten Grundsätzen Geltung zu verschaffen und neu hinzutretende Spendeeinrichtungen in diese Vereinbarung aufzunehmen.

Der Deutsche Städtetag, der Deutsche Städte- und Gemeindebund (Abb. 1) und der Verband der Universitätsklinika Deutschland e.V. (Abb. 2) sind Unterstützer der Vereinbarung.

Die Unterzeichner dieses Beitrags haben als Vertreter der in der Präambel unter 6) genannten Blutspendeeinrichtungen die «Vereinbarung nach $\S 3$ Absatz 2 Satz 3 des Transfusionsgesetzes (TFG) zwischen den Spendeeinrichtungen in der Bundesrepublik Deutschland» entsprechend dem gesetzlichen Auftrag erarbeitet.

\section{Disclosure}

The authors declared no conflict of interest. 